\title{
Vom Wunsch zum Ziel?! Potential von Technologien zur Selbstverbesserung
}

\author{
Sarah Diefenbach, Jasmin Niess
}

Department Psychologie, Ludwig-Maximilians-Universität München

\section{Zusammenfassung}

Immer häufiger werden interaktive Produkte Unterstützer von Selbstverbesserung (z.B. ErnährungsApps, Fitness-Gadgets) und übernehmen damit eine verantwortungsvolle Rolle. Es scheint jedoch, dass Technikgestaltung und Psychologie hier noch nicht optimal verzahnt sind und der "therapeutische Dialog" zwischen Produkt und Nutzer mehr Beachtung finden muss. Anhand einer empirischen Nutzerbefragung ( $\mathrm{N}=62$ ) diskutiert der Beitrag Motivation, Wünsche und Wahrnehmungen in der "Kommunikation" mit interaktiven Technologien und skizziert Aufgaben zukünftiger Forschung.

\section{Einleitung}

Interaktive Produkte können im Alltag auf unterschiedliche Weise Unterstützung bieten: bei praktischen Aufgaben (Wäsche waschen, Toast toasten, Navigation von A nach B usw.), aber auch beim Wunsch nach persönlicher Veränderung und Steigerung des Wohlbefindens (z.B. erhöhte Fitness, gesündere Ernährung, verbessertes Zeitmanagement). Technik wandelt sich vom praktischen Tool zum interaktiven Coach und Berater. Es stellt sich die Frage, wie interaktive Produkte dieser anspruchsvollen Rolle angemessen begegnen können und was es für eine optimale Unterstützung von Veränderungsprozessen zu beachten gilt. Aktuelle Überblicksanalysen (z.B. Conroy et al., 2014; Free et al., 2013) zeigen, dass Psychologie und Technikgestaltung noch nicht optimal verzahnt sind: Psychologisch fundierte Interventionen begnügen sich oft mit einer Übertragung etablierter Programme (z.B. Wochenlektionen aus Selbsthilfe-Ratgebern in Buchform) in ein neues Medium (Online-Training, App). Andere Anwendungen nutzen spezifische Möglichkeiten technischer Produkte vernachlässigen aber die psychologische Perspektive. Beispielsweise beschränken sich Tracking-Funktionalitäten oft auf Dokumentation (drei Nächte schlecht geschlafen), ohne relevante Kontextfaktoren oder Verbesserungsmöglichkeiten aufzuzeigen (ich schlafe besser, wenn ich am Vorabend joggen war). Ähnliche Tendenzen zeigt eine Analyse von 167 verfügbaren Apps mit dem Ziel der Steigerung körperlicher Aktivität (Conroy et al., 2014): Die Apps nutzen insgesamt nur ein eingeschränktes Spektrum von Techniken zur Verhaltensänderung, nur wenige integrieren theoretische Modelle relevanter Disziplinen wie Psychologie und Gesundheitswissenschaften. 
Die Mehrzahl der Apps baut auf erzieherisch-belehrende Ansätze (z.B. Vormachen der korrekten Ausführung von Fitnessübungen), motivationale Ansätze finden kaum Berücksichtigung. Was aus unserer Sicht im Kontext von Selbstverbesserungstechnologien fehlt ist eine Auseinandersetzung mit dem Produkt selbst, welches als "materialisiertes Argument" zwischen Nutzer und Gestalter (Redström, 2006) zum Träger der "therapeutischen Kommunikation" wird.

\section{Nutzerbefragung: Wahrnehmungen und Wünsche im Dialog mit Technologien zur Selbstverbesserung}

In einer explorativen Studie untersuchten wir die Wahrnehmung von bestehenden Technologien zur Selbstverbesserung (z.B. Apps, Fitness-Gadgets) von Nutzerseite: Sind Nutzer sensibel für die Art der "Kommunikation" des Produkts? Welche Faktoren sind relevant für Nutzungsmotivation und -abbruch? Von 80 Personen sind/waren 62 Nutzer von interaktiven Produkten zur Unterstützung von Veränderung und bildeten die finale Stichprobe (46 Frauen, Durchschnittsalter 23 Jahre; $\mathrm{sd}=6,2 ; \min =19 ; \max =65$ ). Bei den genutzten Produkten handelte es sich vorwiegend $(44,71 \%)$ um Smartphone-Apps, andere Beispiele waren Fitness-Armbänder, elektrische Zahnbürsten mit Display oder Lichtwecker (Abb. 1). Die Wünsche nach Selbstverbesserung betrafen die Bereiche Sport (28, 45\%), Ernährung (7, 11\%) oder Finanzmanagement $(6,10 \%)$, weitere Bereiche waren Stressreduktion, Steigerung der Konzentrationsfähigkeit, Optimierung des Schlafverhaltens oder Medikamenteneinnahme.
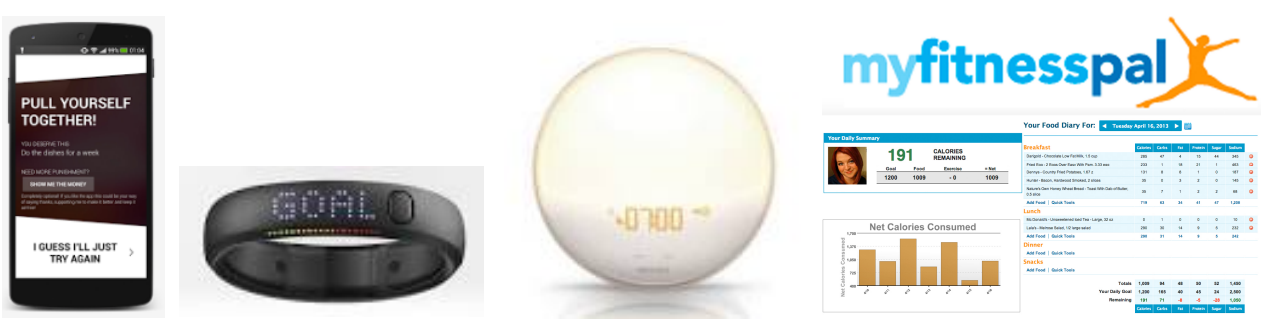

Abbildung 1: Beispiele genutzter Technologien zur Selbstverbesserung

\subsection{Erfolgsnutzer und Abbrecher}

Von den 62 Studienteilnehmern nutzen 33 das Produkt aktuell, 3 Personen beendeten die Nutzung nach erfolgreicher Hilfe des Produkts. Diese 36 Personen werden im Folgenden als "Erfolgsnutzer" bezeichnet. 26 Personen haben die Nutzung abgebrochen, ohne dass das Produkt ihren Veränderungswunsch unterstützen konnte (im Folgenden bezeichnet als "Abbrecher"). Als Gründe nennen diese mit der Nutzung verbundene negative Gefühle ("Produkt ging mir auf die Nerven", "häufig schlechtes Gewissen"), den hohen Zeitaufwand bzw. Vorgaben zur Nutzungsfrequenz ("man musste jeden Tag trainieren, das war mir zu viel") oder allgemein, das Produkt habe ihnen nicht so geholfen, wie sie es sich gewünscht hätten. Relevant für eine 
langfristige Nutzung scheint auch das Ausmaß an Eigeninitiative: Eigens ausgesuchte Produkte sind grundsätzlich mit einer längerfristigen Nutzung assoziiert als ein Beginn der Nutzung auf Initiative anderer hin (z.B. Empfehlung, Geschenk, r=.36*). Hier zeigen sich der hohe Wert von Autonomie im Veränderungsprozess sowie die Gefahr von Reaktanz bezüglich "gut gemeinter Ratschläge". In Anlehnung an bestehende Konzeptionen verschiedener Facetten der Unterstützung von Veränderung (Fogg, 1998) erfragten wir die persönliche Erwartung/Wichtigkeit und tatsächlich erfahrene Unterstützung durch das Produkt jeweils auf einer 7er-Skala. Unter den Abbrechern ergaben sich signifikante Differenzen (d.h. wohl möglich enttäuschte Erwartungen) hinsichtlich der Aspekte "Motivationssteigerung" (6,04 vs. 4,35; T(25)=4,28; $\mathrm{p}<.001)$, "Verbesserungshinweise"(5,31 vs. 3,85; $\mathrm{T}(25)=4,21 ; \mathrm{p}<.001)$ sowie die "Erreichung eines konkreten Ziels" (6,00 vs. 4,88; T(25)=4,16; p<.001). Hinsichtlich der Aspekte "bewussteren Einblick in Verhaltensweisen", "erweitertes Wissen" und "Einblick in langfristige Konsequenzen" ergaben sich keine signifikanten Diskrepanzen. Misserfolg scheint also weniger durch mangelnde Informationen sondern eher durch mangelnde Motivation und Wahrnehmung konkreter Verbesserungsmöglichkeiten erklärbar.

\subsection{Wahrgenommener Charakter und Kommunikationsstil}

Für die Erhebung des wahrgenommenen "Kommunikationsstils" des Produkts entwickelten wir ein Ratinginstrument, welches nach einem etablierten Modell der Kommunikationspsychologie (Schulz von Thun, 1989) acht Stile unterscheidet. Für jede Dimension/Stil vergaben die Teilnehmer ein Rating von 0-3. Abbildung 2 zeigt die Mittelwerte für die acht Dimensionen, die Unterschiede zwischen den Dimensionen sind signifikant $(F(1,50)=480, p<.001)$. Dies zeigt die generelle Beschreibungsfähigkeit und Sensibilität für unterschiedliche Kommunikationsstile, auch im Dialog mit interaktiven Produkten.

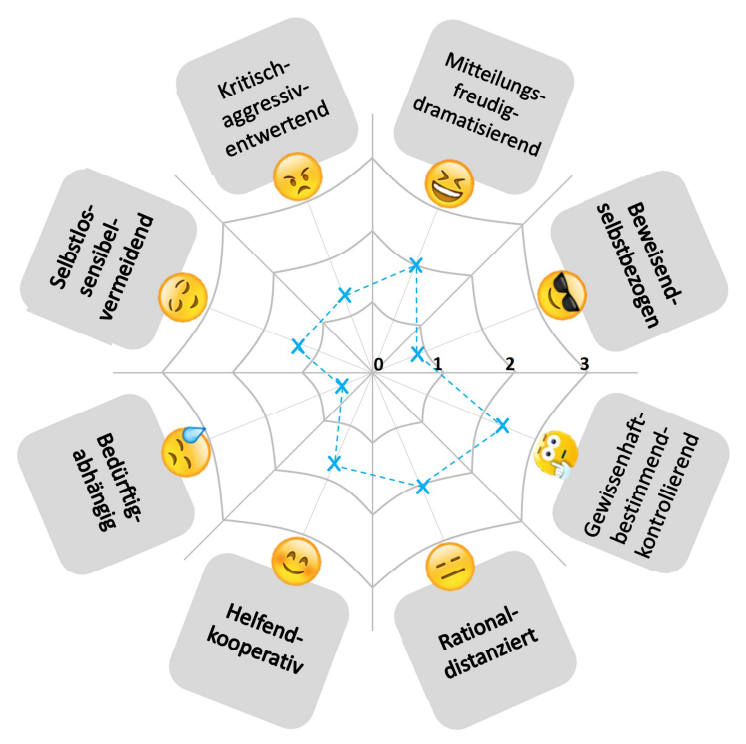

Abbildung 2: Wahrgenommener Kommunikationsstil von Selbstverbesserungstechnologien 
Die Frage nach dem wahrgenommenen "Produktcharakter" lieferte allgemein positive Attribute (cool, süß, sympathisch) sowie positive Attribute zur Unterstützung von Veränderung (hilfreich, unterstützend). Bei Attributen im Zusammenhang mit der Art der Dokumentation, Rückmeldung und Beeinflussung von Verhalten zeigten sich feine Unterschiede zwischen Erfolgsnutzern und Abbrechern: Während Erfolgsnutzer eher positiv-neutrale Adjektiven nannten (analytisch, ehrlich, unaufdringlich, zuverlässig), teils auch Kombinationen, welche den ambivalenten Charakter unbequemer Hinweise wiedergeben (süffisant-mahnend), zeigen Abbrecher eine Verschiebung in Richtung negativ konnotierter Attribute (bestimmend, dominant, mächtig, fordernd). Angesichts des schmalen Grats zwischen Einflussnahme und Bevormundung braucht es genauere Einblicke dazu, welche Gestaltungselemente ein Produkt noch "bitter-süß" oder nur noch "bitter" erscheinen lassen.

\section{Diskussion und Ausblick}

Unsere Befragung zeigt die hohe Nutzungsbereitschaft für Selbstverbesserungstechnologien (62 von 80 Personen, 78\%). Gleichzeitig zeigt der beträchtliche Anteil von Personen mit Abbruch der Nutzung von Zielerreichung (26 von 62, 42\%) verschenktes Potential, welches durch sensiblere, psychologisch-fundierte Gestaltungsentscheidungen besser genutzt werden könnte. Wie schon Fogg (1998, S. 231) betonte, liefert psychologisches Wissen eine wichtige Basis für das Feld Persuasive Technology. Neben der Einflussnahme seitens des Produkts will unsere Forschung auch die Rezeption der Produktbotschaft seitens des Nutzers stärker in den Fokus rücken. Zukünftige Studien sollen weitere Einsichten dazu generieren, wie interaktive Produkte psychologische Expertise im Dialog mit dem Nutzer sinnvoll repräsentieren und eine hilfreiche "therapeutische Haltung" einnehmen können. Mögliche Ansatzpunkte sind hier beispielsweise Timing und Art von Empfehlungen sowie ein positiv konnotiertes Framing von Veränderungsbedarf.

\section{Literaturverzeichnis}

Conroy, D. E., Yang, C. H., \& Maher, J. P. (2014). Behavior change techniques in top-ranked mobile apps for physical activity. American journal of preventive medicine, 46(6), 649-652.

Fogg, B. J. (1998). Persuasive computers: perspectives and research directions. In Proceedings of CHI 1998, ACM Press, 225-232

Free, C., Phillips, G., Galli, L., Watson, L., Felix, L., Edwards, P. \& Haines, A. (2013). The effectiveness of mobile-health technology-based health behaviour change or disease management interventions for health care consumers: a systematic review. PLoS medicine, 10(1), e1001362

Redström, J. (2006), Persuasive design: fringes and foundations. In Persuasive 06, Berlin, Heidelberg: Springer, 112-122.

Schulz von Thun, F. (1989). Miteinander reden 2: Stile, Werte und Persönlichkeitsentwicklung-Differentielle Psychologie der Kommunikation. Reinbek: Rowohlt.

\section{Kontaktinformationen}

Prof. Dr. Sarah Diefenbach, Ludwig-Maximilians-Universität München, sarah.diefenbach@1mu.de 